\title{
Constitutive Relation for Large Deformations of Fiber-Reinforced Rubberlike Materials with Different Response in Tension and Compression
}

\author{
REFERENCE: Li, Q., Dillard, D. A., and Batra, R. C., "Constitutive Relation for Large \\ Deformations of Fiber-Reinforced Rubberlike Materials with Different Response in \\ Tension and Compression," Tire Science and Technology, TSTCA, Vol. 44, No. 1, January- \\ March 2016, pp. 51-72.
}

\begin{abstract}
Fiber-reinforced rubberlike materials commonly used in tires undergo large deformations and exhibit different responses in tension and compression along the fiber direction. Assuming that the response of a fiber-reinforced rubberlike material can be modeled as transversely isotropic with the fiber direction as the axis of transverse isotropy, we express the stored energy function in terms of the five invariants of the right Cauchy-Green strain tensor and account for different response in tension and compression along the fiber direction. The constitutive relation accounts for both material and geometric nonlinearities and incorporates effects of the fifth strain invariant, $I_{5}$. It has been shown by Merodio and Ogden that in shear dominated deformations, $I_{5}$ makes a significant contribution to the stress-strain curve. We have implemented the proposed constitutive relation in the commercial software, LS-DYNA. The numerical solutions of a few boundary value problems studied here agree with their analytical solutions derived by using Ericksen's inverse approach, in which part of the solution is assumed and unknowns in the presumed solution are found by analyzing the pertinent boundary value problem. However, computed results have not been compared with experimental findings. When test data become available, one can modify the form of the strain energy density and replace the proposed constitutive relation by the new one in LS-DYNA.
\end{abstract}

KEY WORDS: fiber-reinforced rubberlike material model, user defined subroutine, finite plane strain bending, different response in tension and compression

\section{Introduction}

Fiber-reinforced rubberlike materials exhibit high specific strength and stiffness in the fiber direction that can be exploited to reduce weight and cost and simultaneously increase durability of components (e.g., tires, belts, seals, and impact absorbing cushions) made of such materials. The rubberlike matrix, with low strength and stiffness but large values of failure strain, maintains fibers in their relative positions, thereby providing desirable mechanical properties. Optimal design of these composites (e.g., fiber volume fraction, fiber orientation, fiber/ matrix bonding, matrix material) requires the development of efficient, robust, and reliable numerical techniques to accurately predict the system level response of

${ }^{1}$ Department of Biomedical Engineering \& Mechanics, M/C 0219, Virginia Polytechnic Institute and State University, Blacksburg, Virginia 24061, USA

${ }^{2}$ Corresponding author. Department of Biomedical Engineering \& Mechanics, M/C 0219, Virginia Polytechnic Institute and State University, Blacksburg, Virginia 24061, USA. Email: rbatra@vt.edu 
structures made of these materials to applied loads, since experimentally designing and characterizing them is time consuming and very expensive.

The often used numerical technique to analyze large deformations of engineering structures is the finite element method (FEM). For example, Batra [1] modeled rubber as a Mooney-Rivlin material and used the FEM to analyze finite plane strain deformations of a rubber-covered roll contacting a rigid roll. This work was subsequently generalized to large deformations of viscoelastic roll covers by Bapat and Batra [2].

Several commercial software packages (e.g., ABAQUS, ANSYS, and LSDYNA) based on FE technology are regularly used in the tire industry. However, simulating large deformations of anisotropic materials that exhibit different response in tension and compression along the fiber direction is quite challenging, and $\mathrm{FE}$ algorithms for large deformations of these materials have not been implemented in many commercial codes. Experimentally validated and thermodynamically consistent constitutive relations are needed for accurately modeling the response of such systems.

Tires for transportation vehicles such as cars, trucks, buses, and tractors are usually made of fiber-reinforced rubberlike materials that are anisotropic, inhomogeneous, viscoelastic, and nearly incompressible. As is well known, modeling separately each constituent of the composite in a real size structure is computationally quite expensive since the ratio of the moduli of the fiber to that of the matrix is very large, necessitating an extremely fine FE mesh near the fiber/ matrix interface. A rebar element method developed and successfully applied to study infinitesimal deformations of reinforced concrete structures has been extended to study finite deformations of fiber-reinforced rubberlike materials $[3,4]$. In this method, either one or several planar fiber layers are embedded in a host three-dimensional, eight-node element made of a rubberlike material. The fiber layer is usually modeled as a four-node membrane. The displacements of the four nodes are derived from the displacements of the eight nodes of the parent element. Thus no additional degrees of freedom are introduced, and the computational cost is kept reasonable. However, the rebar element has had limited success for moderately large strains [5]. In the rebar layer model implemented in ABAQUS [6], the fiber is assumed to be loaded in the axial direction, and the fiber material is assumed to be incompressible and hyperelastic.

The current state of the art is to replace the inhomogeneous material by an equivalent homogeneous material whose mechanical properties depend upon the volume fractions and the mechanical properties of the constituents and analyze the same system level problem with the homogenized material. This approach provides useful information at a reasonable computational cost, especially during the early stages of design. Results derived from the use of the equivalent homogeneous material should be compared with those from experiments to validate the mathematical model. Of course, the use of a homogenized material precludes consideration of stress singularities at the fiber/ 
matrix interface and near fiber ends. Thus the stress distribution in the homogenized material may be quite different from that in the inhomogeneous fiber-reinforced material.

For small deformations, homogenization techniques include the rule of mixtures, the equivalent energy principle, and the Mori-Tanaka scheme. Batra et al. [7], among others, have compared results of different homogenization techniques. However, for large deformations of hyperelastic materials, such as fiber-reinforced rubberlike materials, it is difficult to obtain closed form expressions for the effective properties of the composites due to both material and geometric nonlinearities.

Numerous phenomenological constitutive models have been proposed for fiber-reinforced rubberlike materials that describe well some aspects of experimentally measured material response [8-17]. Pipkin [8] analytically solved several boundary value problems for incompressible fiber-reinforced materials by assuming that the fibers are inextensible. As has been postulated by several authors [9-17], we assume that a fiber-reinforced rubberlike material can be modeled as transversely isotropic and hyperelastic with the fiber direction as the axis of transverse isotropy. Using the concepts of material objectivity and material symmetry, Ericksen and Rivlin [18] showed that the strain energy density for these materials is at most a function of five invariants of the strain tensor. Batra [19] derived universal relations for these materials that enable one to check whether the material being studied is transversely isotropic.

A challenge is to find an explicit expression for the strain energy density in terms of the five invariants. We recall that the reinforcing fibers generally exhibit different response in tension and compression. Motivated by the success of the Mooney-Rivlin form of the strain energy density for isotropic incompressible rubberlike materials and the works of Pipkin [8], Qiu and Pence [10], and Merodio and Ogden [11], we postulate an expression for the strain energy density in terms of the five invariants of the strain tensor that exhibits different response in tension and compression. For incompressible fiber-reinforced materials, we analytically analyze two homogeneous deformations, namely, the uniaxial tension/compression and simple shear. These illustrate different responses in tension and compression and the effect of including the fifth strain invariant in the constitutive relation. We then study the finite plane strain bending deformations of a rectangular beam into a circular arc for which deformations are inhomogeneous. This problem is a member of Ericksen's family of controllable deformations that can be produced in every isotropic, homogeneous, and incompressible body by only applying surface tractions to the bounding surfaces. Since the beam material is transversely isotropic, Ericksen's theorem is not applicable to this problem. It is shown that the problem can be analytically solved only when fibers are either along the beam axis or along the beam thickness direction. These analytical solutions are compared with the numerical results computed by using LS-DYNA in which this material model has been implemented as a user defined subroutine. The two sets of results are 
found to agree well with each other, thereby lending credibility to the implementation of the material model in the subroutine. In order to fully verify the implementation of the subroutine, several initial boundary value problems need to be analyzed and their numerical solutions compared with the analytical solutions. One could potentially use the method of manufactured solutions (e.g., see the material just preceding and immediately following Eq. (20) of Batra and Liang [20]; details of the method are better described in section 3.2 of Love and Batra [21] ) to verify the implementation of the material model in the software.

The identification of material parameters in the proposed constitutive relation and their evaluation from test data will be addressed in a future work.

\section{Constitutive Relations for Fiber-Reinforced Rubberlike Materials}

We assume that a fiber-reinforced rubberlike material can be modeled as a homogeneous, transversely isotropic, and hyperelastic with the fiber direction as the axis of transverse isotropy. We use the theory of mixtures (e.g., see Bowen [22]) in which it is assumed that a spatial point is simultaneously occupied by all constituents of a body and they experience the same deformation gradient. The strain energy density at a point equals the sum of the strain energy densities of the constituents occupying that point weighted by their volume fractions. Following the work of Ericksen and Rivlin [18], we express the stored energy density per unit volume in the reference configuration, $W$, in terms of the five invariants of the right Cauchy-Green strain tensor $\boldsymbol{C}$. That is,

$$
W=W\left(I_{1}, I_{2}, I_{3}, I_{4}, I_{5}\right)
$$

where

$$
\begin{aligned}
& I_{1}=\operatorname{tr} \boldsymbol{C}, I_{2}=\frac{1}{2}\left[(\operatorname{tr} \boldsymbol{C})^{2}-\operatorname{tr}\left(\boldsymbol{C}^{2}\right)\right], I_{3}=\operatorname{det} \boldsymbol{C}, \\
& I_{4}=\boldsymbol{A}_{\mathbf{0}} \cdot \boldsymbol{C} \cdot \boldsymbol{A}_{\mathbf{0}}, I_{5}=\boldsymbol{A}_{\mathbf{0}} \cdot \boldsymbol{C}^{2} \cdot \boldsymbol{A}_{\mathbf{0}}
\end{aligned}
$$

In Eqs. (1) and (2), $\boldsymbol{C}=\boldsymbol{F}^{\boldsymbol{T}} \boldsymbol{F}, \boldsymbol{F}=\partial \boldsymbol{x} / \partial \boldsymbol{X}$ is the deformation gradient, and $\boldsymbol{x}$ the position vector in the current or the deformed configuration, with respect to rectangular Cartesian coordinates, of a material point that occupied the place $\boldsymbol{X}$ in the undeformed or the reference configuration. Furthermore, $\boldsymbol{A}_{0}$ is a unit vector along the fiber in the reference configuration, and $I_{1}, I_{2}, I_{3}, I_{4}$, and $I_{5}$ are invariants of the right Cauchy-Green strain tensor for a transversely isotropic material with $\boldsymbol{A}_{0}$ as the axis of transverse isotropy. The invariants $I_{4}$ and $I_{5}$ are related to the stretch along the fiber, and $I_{5}$ also accounts for shearing deformations of the material [11].

For nearly incompressible materials, $W$ is usually expressed as the sum of two terms, one for the volumetric and the other for the distortional deformations. That is, 


$$
W=U(J)+W\left(\bar{I}_{1}, \bar{I}_{2}, \bar{I}_{4}, \bar{I}_{5}\right)
$$

where $J=\operatorname{det}(\boldsymbol{F})=I_{3}^{1 / 2}$ is the ratio of the volume of a material element in the current configuration to that in the reference configuration, and

$$
\bar{I}_{1}=J^{-2 / 3} I_{1}, \bar{I}_{2}=J^{-4 / 3} I_{2}, \bar{I}_{4}=J^{-2 / 3} I_{4}, \bar{I}_{5}=J^{-4 / 3} I_{5}
$$

The deformation gradient $\boldsymbol{F}$ can be written as the product of two matrices [23]

$$
\boldsymbol{F}=\boldsymbol{F}_{\text {vol }} \overline{\boldsymbol{F}}, \boldsymbol{F}_{\text {vol }}=J^{1 / 3} \mathbf{1}, \overline{\boldsymbol{F}}=J^{-1 / 3} \boldsymbol{F}
$$

where $\mathbf{1}$ is the identity matrix. We note that

$$
\operatorname{det}\left[\boldsymbol{F}_{\text {vol }}\right]=J, \operatorname{det}[\overline{\boldsymbol{F}}]=1
$$

Thus $\boldsymbol{F}_{\text {vol }}$ measures changes in volume, and $\overline{\boldsymbol{F}}$ measures distortional deformations. Similarly, the right and the left Cauchy-Green tensor $\boldsymbol{C}$ and $\boldsymbol{B}$, respectively, can be written as

$$
\boldsymbol{C}=\boldsymbol{F}^{\boldsymbol{T}} \boldsymbol{F}=J^{2 / 3} \overline{\boldsymbol{C}}, \boldsymbol{B}=\boldsymbol{F} \boldsymbol{F}^{\boldsymbol{T}}=J^{2 / 3} \overline{\boldsymbol{B}}
$$

For an incompressible material, $J=1$, and $\overline{I_{1}}, \overline{I_{2}}, \overline{I_{4}}$, and $\overline{I_{5}}$ equal, respectively, $I_{1}$, $I_{2}, I_{4}$, and $I_{5}$.

In order to find the explicit form of $W$ for the material of interest, one needs test data from numerous experiments in which one of the invariants is varied and the remaining invariants are kept constant. In the absence of such data, one either expands $W$ in terms of a finite Taylor series or postulates an expression for it that has a few material parameters. The Mooney and the Mooney-Rivlin material models can be regarded as examples of $W$ expanded in finite Taylor series with one and two material constants, respectively. We note that Mooney derived the expression for $W$ by using the kinetic theory of rubber [24]. Here we assume a simple expression for $W$ with the understanding that if predictions from it do not agree well with the test data then the expression can be modified.

For incompressible and nearly incompressible fiber-reinforced rubberlike materials, we postulate, respectively, Eqs. (8) and (9) for $W$.

$$
\begin{aligned}
& W=\left\{\begin{array}{c}
\left(1-V_{f}\right)\left[C_{1}\left(I_{1}-3\right)+C_{2}\left(I_{2}-3\right)\right], I_{4}<1 \\
\left(1-V_{f}\right)\left[C_{1}\left(I_{1}-3\right)+C_{2}\left(I_{2}-3\right)\right]+V_{f}\left[\gamma_{4}\left(I_{4}-1\right)^{2}+\gamma_{5}\left(I_{5}-1\right)^{2}\right], I_{4} \geq 1
\end{array}\right. \\
& W=\left\{\begin{array}{c}
\frac{K}{2}\left(\frac{J^{2}-1}{2}-\ln J\right)+\left(1-V_{f}\right)\left[C_{1}\left(\bar{I}_{1}-3\right)+C_{2}\left(\bar{I}_{2}-3\right)\right], \bar{I}_{4}<1 \\
\frac{K}{2}\left(\frac{J^{2}-1}{2}-\ln J\right)+\left(1-V_{f}\right)\left[C_{1}\left(\bar{I}_{1}-3\right)+C_{2}\left(\bar{I}_{2}-3\right)\right]+V_{f}\left[\gamma_{4}\left(\bar{I}_{4}-1\right)^{2}+\gamma_{5}\left(\bar{I}_{5}-1\right)^{2}\right], \bar{I}_{4} \geq 1
\end{array}\right.
\end{aligned}
$$


Here $V_{f}$ equals the volume fraction of fibers, and $C_{1}, C_{2}, K, \gamma_{4}$, and $\gamma_{5}$ are material constants whose values are to be determined from test data. Equations (8) and (9) imply that the axial compression of fibers does not contribute to the strain energy density. Thus the problem of finding $W$ has been reduced to that of ascertaining values of material constants. The material parameter $K$ is usually called the bulk modulus and is assigned a value much larger than that of the other four material parameters.

Recalling (e.g., see Batra [23] and Truesdell and Noll [25]) that the second Piola-Kirchhoff stress tensor $S$ is related to the strain energy density function $W$ by

$$
\boldsymbol{S}=2 \frac{\partial W}{\partial \boldsymbol{C}}
$$

and the Cauchy stress tensor $\boldsymbol{\sigma}$ to $S$ by

$$
\sigma=J^{-1} F S F^{T}
$$

we get Eqs. (12) and (13), respectively, for incompressible and nearly incompressible materials.

$$
\begin{aligned}
\boldsymbol{\sigma}= & -p \mathbf{1}+2\left(1-V_{f}\right)\left[C_{1} \boldsymbol{B}+C_{2}\left(I_{1} \boldsymbol{B}-\boldsymbol{B}^{2}\right)\right] \\
& +4 V_{f} H\left(I_{4}-1\right)\left[\gamma_{4}\left(I_{4}-1\right) \boldsymbol{a} \otimes \boldsymbol{a}+\gamma_{5}\left(I_{5}-1\right)(\boldsymbol{a} \otimes \boldsymbol{B} \cdot \boldsymbol{a}+\boldsymbol{B} \cdot \boldsymbol{a} \otimes \boldsymbol{a})\right] \\
J \boldsymbol{\sigma}= & \frac{K}{2}\left[J^{2}-1\right] \mathbf{1}+2\left(1-V_{f}\right)\left[\boldsymbol{C}_{1}\left(\overline{\boldsymbol{B}}-\frac{1}{3} \bar{I}_{1} \mathbf{1}\right)+C_{2}\left(\bar{I}_{1} \overline{\boldsymbol{B}}-\overline{\boldsymbol{B}}^{2}-\frac{2}{3} \bar{I}_{2} \mathbf{1}\right)\right] \\
& +4 V_{f} H\left(\bar{I}_{4}-1\right)\left[\gamma_{4}\left(\bar{I}_{4}-1\right)\left(\overline{\boldsymbol{a}} \otimes \overline{\boldsymbol{a}}-\frac{1}{3} \bar{I}_{4} \mathbf{1}\right)+\gamma_{5}\left(\bar{I}_{5}-1\right)\left(\overline{\boldsymbol{a}} \otimes \overline{\boldsymbol{B}} \cdot \overline{\boldsymbol{a}}+\overline{\boldsymbol{B}} \cdot \overline{\boldsymbol{a}} \otimes \overline{\boldsymbol{a}}-\frac{2}{3} \bar{I}_{5} \mathbf{1}\right)\right]
\end{aligned}
$$

Here $H$ is the Heaviside function, $\boldsymbol{a} \otimes \boldsymbol{b}$ denotes the tensor product between vectors $\boldsymbol{a}$ and $\boldsymbol{b}, \boldsymbol{a}$ and $\overline{\boldsymbol{a}}$ are unit vectors along the vector $\boldsymbol{F} \boldsymbol{A}_{0}$, and the hydrostatic pressure $p$ is not determined from the deformation gradient but is found by solving the pertinent initial boundary value problem, provided that normal tractions are prescribed on a part of the boundary. Thus for an incompressible material one needs to find three components of displacement and the hydrostatic pressure, whereas for a compressible material only three displacement components need to be determined. For axial compression along the fibers, the term multiplying $V_{f}$ does not contribute to the Cauchy stress, resulting in a discontinuous slope at zero strain of the axial stress-axial strain curve for uniaxial deformations along the fiber. Thus the speed of longitudinal waves along the fiber direction will depend upon whether fibers are being compressed or stretched. Modifications of the constitutive relation to overcome this are being considered. 
We note that most FE-based commercial solvers do not regard the hydrostatic pressure in incompressible materials as an independent variable, assume the material to be nearly incompressible, and approximate incompressibility by assigning a very large value to the bulk modulus that serves as a penalty parameter. Batra [1], among others, used a mixed formulation in which the pressure field is taken as an independent variable.

\section{Implementation of the Material Model in LS-DYNA}

The commercial software LS-DYNA [26] is based on the FE technology for analyzing transient deformations of structures made of different materials. We have implemented the material model described in the section "Constitutive Relations for Fiber-Reinforced Rubberlike Materials" above as a user defined subroutine, written in FORTRAN, that employs eight-node brick elements and the constitutive Eqs. (12) and (13) to analyze finite deformations of fiber-reinforced rubberlike materials. In order to use the subroutine, one needs, in addition to the regular input parameters required to analyze a problem by the FEM, values of the volume fraction of fibers, $V_{f}$, components of the unit vector $\boldsymbol{A}_{0}$ defining the fiber orientation in the reference configuration, and values of the material parameters appearing in the constitutive relation [Eq. (13)].

\section{Verification of the Implementation of the User Defined Subroutine}

We analytically and numerically study a few boundary value problems for fiber-reinforced materials and compare the two solutions to verify the subroutine developed and implemented in LS-DYNA. For the first two problems analyzed, namely, simple extension/compression and simple shear, deformations are homogeneous. Thus the deformation gradient $\boldsymbol{F}$ and hence stresses are constants in a homogeneous body, and the balance of linear momentum with zero body and null inertia forces is trivially satisfied. This implies that a FE mesh with only one element should suffice to numerically analyze the problem. The third problem involves bending of a straight beam into a circular arc in which the deformation gradient $\boldsymbol{F}$ varies throughout the body. This problem has been numerically studied with LS-DYNA using mass scaling, i.e., artificially reducing the mass density to increase the wave speed. The element matrices have been computed by using the one-point integration rule and Belytschko-Bindeman hourglass control [27]. While checking the energy balance, the kinetic energy and the hourglass mode energies have been found to be negligible, ensuring that static problems have been studied and the total energy is conserved.

While analytically analyzing a boundary value problem, the material of the body is assumed to be incompressible. However, during the numerical solution of the same boundary value problem, the material is taken to be nearly 
incompressible since the FE technology implemented in LS-DYNA does not allow the assumption of perfect incompressibility. For sufficiently large values of $K$, the solution of the boundary value problem for nearly incompressible materials is very close to that for incompressible materials. We note that values of material parameters are not critical for verifying the implementation of the subroutine. Thus we have set $\gamma_{4}=\gamma_{5}=1000\left(C_{1}+C_{2}\right), C_{1}=C_{2}=1 \mathrm{MPa}$, and the volume fraction of fibers $=0.3$. The bulk modulus, $K$, is assigned a very large value (e.g., 250,000 $\gamma_{4}$ ) to mimic the nearly incompressible response of the homogenized material for simple extension/compression and simple shear deformations. However, for the plane strain bending problem analyzed, the bulk modulus is set equal to $150 \gamma_{4}$, since a larger value of $K$ required more than 65 central processing unit (CPU) hours on 12 processors. Thus the incompressibility condition for the bending problem is not well satisfied.

\section{Simple Extension of a Cube}

We study simple extensional deformations of a cube of edge length $L$ with equal and opposite axial surface tractions applied on the surfaces $x_{1}=X_{1}=0$ and $L$, and fibers oriented along the vector $\boldsymbol{A}_{0}=(\cos \alpha, \sin \alpha, 0)$. That is, fibers are in the $X_{1} X_{2}$-plane and are inclined at an angle $\alpha$ to the $X_{1}$-axis. A schematic sketch of the problem studied is shown in Fig. 1. While numerically solving the problem, the body is deformed by setting $u_{1}=x_{1}-X_{1}=0$ for nodes on the surface $X_{1}=0, u_{2}=x_{2}-X_{2}=0$ for nodes on the surface $X_{2}=0, u_{3}=0$ at one node on the surface $X_{1}=0$, and simultaneously prescribing uniform values of $u_{1}$ and $u_{2}$ on the surfaces $X_{1}=L$, and $X_{2}=L$. The rigid body translation is eliminated by setting $u_{3}=0$ at a node on the surface $X_{1}=0$. The nonlinear problem is solved by dividing all displacements into 100 equal parts and simultaneously incrementing every displacement component by $1 \%$. The surfaces $X_{3}=$ constant are kept traction free.

Assuming that deformations are homogeneous, we can write the deformation field as

$$
x_{1}=\lambda_{1} X_{1} ; x_{2}=\lambda_{2} X_{2} ; x_{3}=\lambda_{3} X_{3}
$$

where $\lambda_{1}, \lambda_{2}$, and $\lambda_{3}$ are stretches along the $X_{1}, X_{2}$, and $X_{3}$ coordinate axes, respectively. For the deformation [Eq. (14)] to be admissible in an incompressible material, $\lambda_{3}=1 / \lambda_{1} \lambda_{2}$.

For the deformation field [Eq. (14)], the strain invariants have the following expressions:

$$
\begin{aligned}
& I_{1}=\lambda_{1}^{2}+\lambda_{2}^{2}+\lambda_{1}^{-2} \lambda_{2}^{-2} \\
& I_{2}=\lambda_{1}^{-2}+\lambda_{2}^{-2}+\lambda_{1}^{2} \lambda_{2}^{2}, I_{3}=1 \\
& I_{4}=(\cos \alpha)^{2} \lambda_{1}^{2}+(\sin \alpha)^{2} \lambda_{2}^{2} \\
& I_{5}=(\cos \alpha)^{2} \lambda_{1}^{4}+(\sin \alpha)^{2} \lambda_{2}^{4}
\end{aligned}
$$



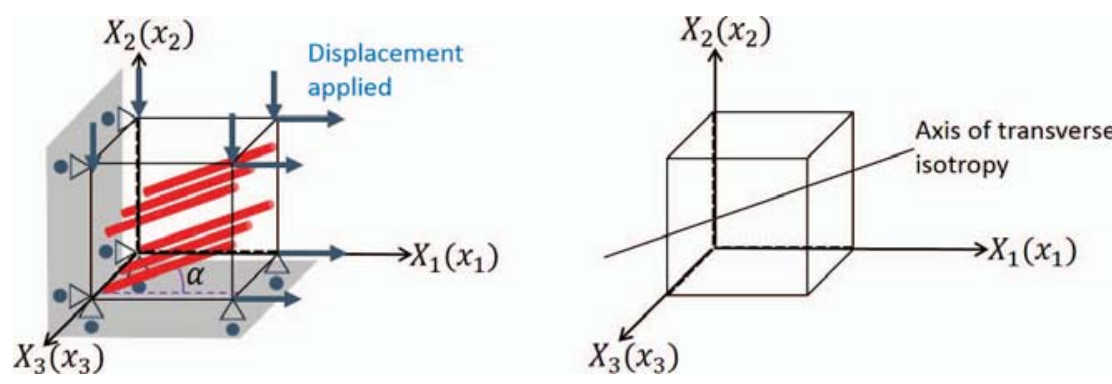

FIG. 1 - Schematic sketch of the simple extension of a cube made of a fiber-reinforced rubberlike material with fibers inclined at angle $\alpha$ to the $\mathrm{X}_{1} \mathrm{X}_{3}$ - plane; (left) fiber-reinforced body, (right) equivalent homogenized transversely isotropic body.

Substituting for $I_{1}, I_{2}, I_{4}$, and $I_{5}$ from Eqs. (14)-(15) into Eq. (12), we get the following expressions for components of the Cauchy stress tensor.

$$
\begin{aligned}
\sigma_{11}= & -p+2\left(1-V_{f}\right)\left[C_{1} \lambda_{1}^{2}+C_{2} \lambda_{1}^{2}\left(\lambda_{2}^{2}+\lambda_{3}^{2}\right)\right] \\
& +4 V_{f} H\left(I_{4}-1\right)\left[\gamma_{4}\left(I_{4}-1\right)(\cos \alpha)^{2} \lambda_{1}^{2}+2 \gamma_{5}\left(I_{5}-1\right)(\cos \alpha)^{2} \lambda_{1}^{4}\right] \\
\sigma_{22}= & -p+2\left(1-V_{f}\right)\left[C_{1} \lambda_{2}^{2}+C_{2} \lambda_{2}^{2}\left(\lambda_{1}^{2}+\lambda_{3}^{2}\right)\right] \\
& +4 V_{f} H\left(I_{4}-1\right)\left[\gamma_{4}\left(I_{4}-1\right)(\sin \alpha)^{2} \lambda_{2}^{2}+2 \gamma_{5}\left(I_{5}-1\right)(\sin \alpha)^{2} \lambda_{2}^{4}\right] \\
\sigma_{33}= & -p+2\left(1-V_{f}\right)\left[C_{1} \lambda_{3}^{2}+C_{2} \lambda_{3}^{2}\left(\lambda_{1}^{2}+\lambda_{2}^{2}\right)\right] \\
\sigma_{12}= & \sigma_{21}=2 V_{f} \lambda_{1} \lambda_{2} \sin 2 \alpha H\left(I_{4}-1\right)\left[\gamma_{4}\left(I_{4}-1\right)+\gamma_{5}\left(I_{5}-1\right)\left(\lambda_{2}^{2}+\lambda_{1}^{2}\right)\right] \\
\sigma_{13}= & \sigma_{31}=\sigma_{23}=0
\end{aligned}
$$

Equilibrium equations require that the hydrostatic pressure $p$ be a constant. We assume that the normal traction on the lateral surface $\mathrm{x}_{3}=0, \mathrm{~L}$ vanishes. Thus $\sigma_{33}=0$, and

$$
p=2\left(1-V_{f}\right)\left[C_{1} \lambda_{3}^{2}+C_{2}\left(\lambda_{3}^{2} \lambda_{1}^{2}+\lambda_{3}^{2} \lambda_{2}^{2}\right)\right]
$$

Substituting for $p$ from Eq. (17) into Eq. (16), and using the boundary condition $\sigma_{22}=0$ gives

$$
\begin{aligned}
0= & 2\left(1-V_{f}\right)\left\{\left(\lambda_{2}^{2}-\frac{1}{\lambda_{1}^{2} \lambda_{2}^{2}}\right)\left(C_{1}-C_{2} \lambda_{1}^{2}\right)\right\} \\
& +4 V_{f} H\left(I_{4}-1\right)\left[\gamma_{4}\left(I_{4}-1\right)(\sin \alpha)^{2} \lambda_{2}^{2}+2 \gamma_{5}\left(I_{5}-1\right)(\sin \alpha)^{2} \lambda_{2}^{4}\right]
\end{aligned}
$$

Thus the nonzero components of the Cauchy stress are given by 


$$
\begin{aligned}
\sigma_{11}= & 2\left(1-V_{f}\right)\left[C_{1}\left(\lambda_{1}^{2}-\frac{1}{\lambda_{1}^{2} \lambda_{2}^{2}}\right)+C_{2}\left(\lambda_{2}^{2} \lambda_{1}^{2}-\frac{1}{\lambda_{1}^{2}}\right)\right] \\
& +4 V_{f} H\left(I_{4}-1\right)\left[\gamma_{4}\left(I_{4}-1\right)(\cos \alpha)^{2} \lambda_{1}^{2}+2 \gamma_{5}\left(I_{5}-1\right)(\cos \alpha)^{2} \lambda_{1}^{4}\right], \\
\sigma_{12}= & \sigma_{21}=2 V_{f} \lambda_{1} \lambda_{2} \sin 2 \alpha H\left(I_{4}-1\right)\left[\gamma_{4}\left(I_{4}-1\right)+\gamma_{5}\left(I_{5}-1\right)\left(\lambda_{2}^{2}+\lambda_{1}^{2}\right)\right]
\end{aligned}
$$

with the value of $\lambda_{2}$ expressed as a function of $\lambda_{1}$ by solving Eq. (18).

Except for $\alpha=0$ and $\alpha=90^{\circ}$, besides the axial stress $\sigma_{11}$ one must also apply tangential tractions $\sigma_{12}$ on the bounding surfaces, $x_{1}=$ constant and $x_{2}=$ constant, to produce simple extensional deformations in a fiber-reinforced rubberlike material. The magnitude of surface tractions also depends upon $I_{4}$ and $I_{5}$.

Because of the difficulty in applying deformation dependent surface tractions in LS-DYNA, while numerically solving the problem we gradually increased the above mentioned applied displacements on surfaces $X_{1}=L$ and $X_{2}$ $=L$ of the block while keeping surfaces $X_{3}=0, L$ traction free.

For $\alpha=0^{\circ}, 30^{\circ}$, and $90^{\circ}$ the computed axial stress-axial stretch curves are compared in Fig. 2 with the corresponding analytical ones. We note that $\alpha=$ $0^{\circ}, \lambda_{1}<1$ for axial compression and $\lambda_{1}>1$ for axial tension along the fibers. For $\lambda_{1}$ varying from 0.5 to 2 , stresses computed from the user defined subroutine agree very well with their corresponding analytical values, with the maximum difference between the two being $0.03 \%$. For each case, one can clearly see the change in the slope of the axial stress vs. the axial strain curve at $\lambda_{1}=1$ due to the different response in axial tension and axial compression along the fibers. For $\alpha=30^{\circ}$ tangential tractions are also needed on the planes $X_{2}=$ constant and $X_{1}=$ constant to produce the deformation field given by Eq. (14). For $\alpha=90^{\circ}$ the axial traction required on the plane $X_{1}=$ constant is negligible as compared with that for $\alpha=0^{\circ}$, since in the former case essentially the matrix (rubber) is being deformed and fibers are in axial compression due to Poisson's effect.

\section{Simple Shear Deformations of a Cube}

A simple shear deformation of a cube made of a fiber-reinforced material with fibers making an angle $\alpha$ to the $X_{1} X_{3}$-plane is schematically depicted in Fig. 3. It can be represented as

$$
x_{1}=X_{1}+k X_{2} ; x_{2}=X_{2} ; x_{3}=X_{3}
$$

where $k$ can be interpreted as the shear strain. This deformation is isochoric or volume preserving and thus is admissible in a body made of an incompressible material.

For the deformation given by Eq. (20) 

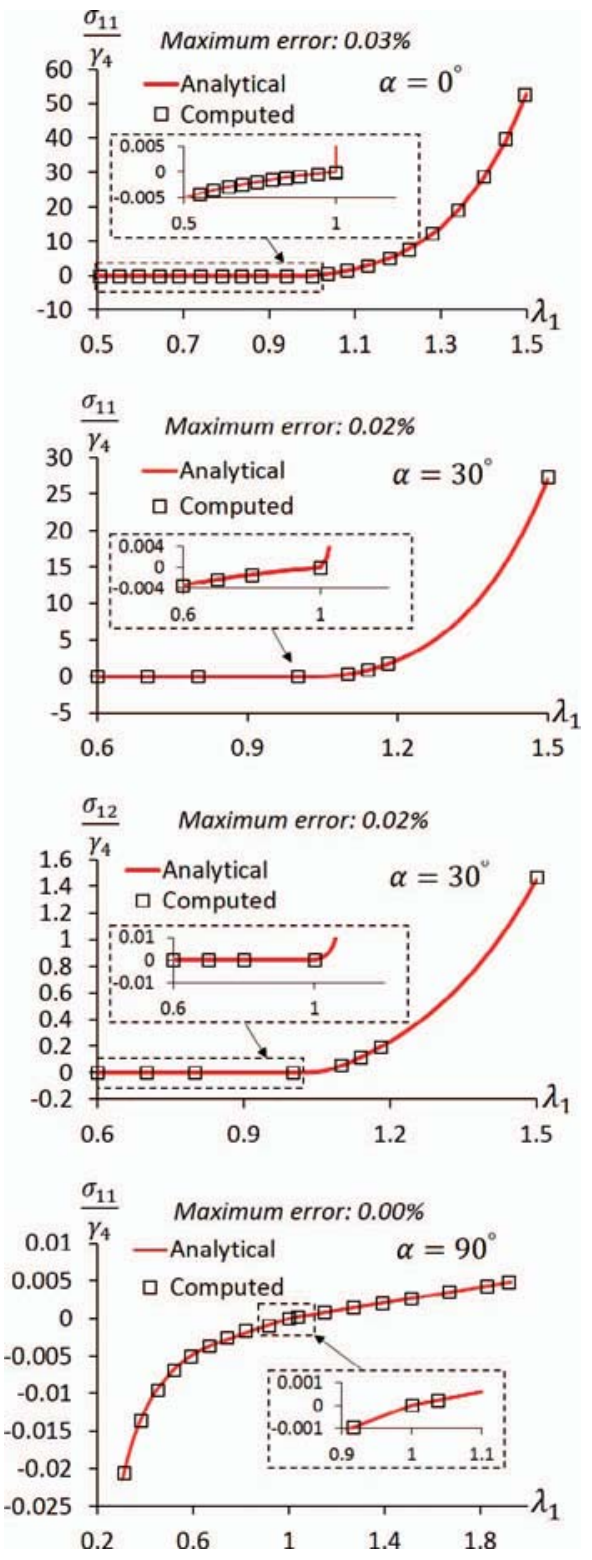

FIG. 2 - Normalized Cauchy stress component vs. the axial stretch for $\alpha=0^{\circ}, 30^{\circ}$, and $90^{\circ}$. 


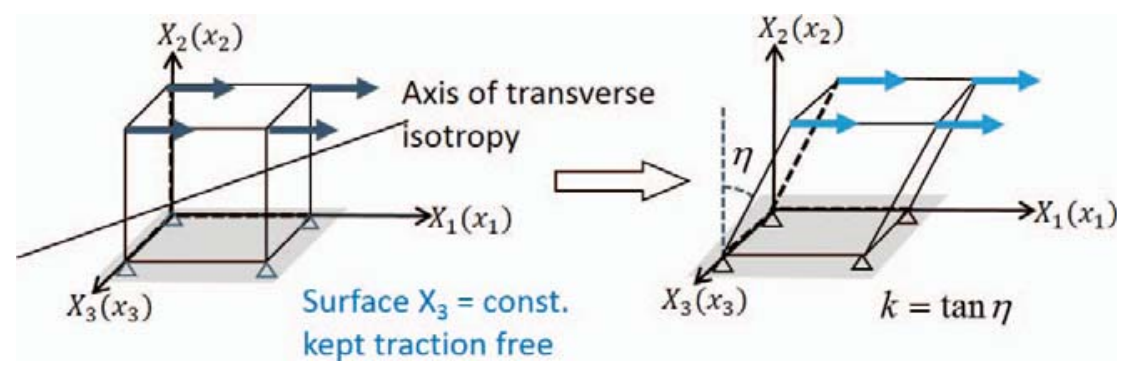

FIG. 3 - Schematic sketch of the simple shearing of a cube made of a fiber-reinforced rubberlike material with fibers inclined at angle $\alpha$ to the $\mathrm{X}_{1} \mathrm{X}_{3}$-plane.

$$
\begin{aligned}
{[B] } & =\left[\begin{array}{ccc}
1+k^{2} & k & 0 \\
k & 1 & 0 \\
0 & 0 & 1
\end{array}\right],[C]=\left[\begin{array}{ccc}
1 & k & 0 \\
k & 1+k^{2} & 0 \\
0 & 0 & 1
\end{array}\right] \\
I_{1} & =k^{2}+3, I_{2}=k^{2}+3, I_{3}=1, \\
I_{4} & =(\sin \alpha)^{2} k^{2}+(\sin 2 \alpha) k+1, \\
I_{5} & =(\cos \alpha)^{2}\left(1+k^{2}\right)+\left[k^{4}+3 k^{2}+1\right](\sin \alpha)^{2}+(\sin 2 \alpha) k\left(2+k^{2}\right)
\end{aligned}
$$

We note that $I_{4}$ depends upon $k$ only when $\alpha \neq 0$. However, $I_{5}$ depends upon $k$ for all values of $\alpha$. Substituting for $I_{1}, I_{2}, I_{4}$, and $I_{5}$ from Eq. (21) into Eq. (12), we arrive at the following expressions for the components of the Cauchy stress tensor.

$$
\begin{aligned}
& \sigma_{11}=-p+2\left(1-V_{f}\right)\left[C_{1}\left(k^{2}+1\right)+C_{2}\left(k^{2}+2\right)\right]+4 V_{f} H\left(I_{4}-1\right)\left\{\gamma_{4}\left(I_{4}-1\right)(\cos \alpha+k \sin \alpha)^{2}+2 \gamma_{5}\left(I_{5}-1\right)\right. \\
&\left.\times\left[\left(k^{4}+2 k^{2}\right)(\sin \alpha)^{2}+\left(1+k^{2}\right)(\cos \alpha)^{2}+\left(3 k+2 k^{3}\right) \cos \alpha \sin \alpha\right]\right\} \\
& \sigma_{22}=-p+2\left(1-V_{f}\right)\left(C_{1}+2 C_{2}\right)+4 V_{f} H\left(I_{4}-1\right)\left\{\gamma_{4}\left(I_{4}-1\right)(\sin \alpha)^{2}+2 \gamma_{5}\left(I_{5}-1\right)\left[(\sin \alpha)^{2}+(\sin \alpha)^{2} k^{2}+k \cos \alpha \sin \alpha\right]\right\} \\
& \sigma_{33}=-p+2\left(1-V_{f}\right)\left[C_{1}+C_{2}\left(k^{2}+2\right)\right] \\
& \sigma_{12}=\sigma_{21}=2\left(1-V_{f}\right)\left(C_{1}+C_{2}\right) k+4 V_{f} H\left(I_{4}-1\right) \\
& \quad\left\{\gamma_{4}\left(I_{4}-1\right)\left[\cos \alpha \sin \alpha+(\sin \alpha)^{2} k\right]+\gamma_{5}\left(I_{5}-1\right)\right. \\
&\left.\quad \times\left[\left(2 k^{3}+2 k\right)(\sin \alpha)^{2}+\cos \alpha \sin \alpha\left(3 k^{2}+2\right)+k\right]\right\} \\
& \sigma_{13}=\sigma_{31}=\sigma_{32}=\sigma_{23}=0
\end{aligned}
$$

We assume that surfaces $x_{3}=X_{3}=$ constant are traction free. Thus $\sigma_{13}=\sigma_{33}=\sigma_{23}$ $=0$, and we get

$$
p=2\left(1-V_{f}\right)\left[C_{1}+C_{2}\left(k^{2}+2\right)\right]
$$


and all components of the Cauchy stress tensor can be evaluated from the known values of the shear strain $k$ and the material parameters.

For $V_{f}=0$, the shear stress $\sigma_{12}$ is proportional to the shear strain $k$ with $2\left(C_{1}+C_{2}\right)$ equaling the shear modulus. The difference between the infinitesimal and the finite deformation problems is that in the former case no normal tractions on the flat bounding surfaces act, while for the latter normal tractions $\sigma_{11}$ and $\sigma_{22}$ given by Eq. (22) are needed to keep the bounding surfaces flat. This is usually referred to as the Poynting effect; e.g., see Truesdell and Noll [25]. Note that $\sigma_{11}$ and $\sigma_{22}$ are not equal to each other in general, implying thereby that different normal surface tractions are needed on the faces $X_{1}=0, L$ and $X_{2}=0, L$ to maintain simple shearing deformations of the block.

For $V_{f} \neq 0$, the three stress components $\sigma_{11}, \sigma_{12}$, and $\sigma_{22}$ depend upon the shear strain $k$ and the angle $\alpha$.

While numerically analyzing simple shearing deformations of a cube of the fiber-reinforced material, we keep faces $x_{3}=X_{3}=$ constant traction free and apply incremental displacements $u_{1}$ and $u_{2}$ given by Eq. (20) to the remaining four faces by following the same procedure as that for the simple tension/compression problem. For $\alpha=0^{\circ}, 30^{\circ}$, and $90^{\circ}$, the computed and the analytical values of normalized stress components for values of the shear strain $k$ between 0 and 1 are compared in Fig. 4. For the range $[0,1]$ of values of $k$ considered, the Cauchy stresses are monotonically increasing functions of the shear strain $k$. The computed and the analytical solutions differ from each other by at most $0.0292 \%$, thereby verifying the subroutine for simple shearing deformations. For $\alpha=0^{\circ}$, i.e., fibers along the $X_{1}$-axis in the reference configuration, $I_{4}=1$ but $I_{5}=\left(1+k^{2}\right)$. Thus for $\alpha=0^{\circ}$ the presence of fibers affects shear stresses only if $W$ depends upon $I_{5}$. For $\alpha=30^{\circ}$, as depicted in Fig. 4, the slope of $\sigma_{12}$ versus $k$ curve at $k=0$ is different for $k>0$ and $k<0$ because of the assumed form of the strain energy density function. For $\alpha=90^{\circ}$ both $I_{4}$ and $I_{5}$ contribute to $W, \sigma_{11}, \sigma_{12}$, and $\sigma_{22}$.

Plane Strain Bending of Fiber-Reinforced Rectangular Beam

For a rectangular beam made of a homogeneous, isotropic, and incompressible hyperelastic material, Ericksen [28] showed that a straight beam can be bent into a circular arc by applying the needed surface tractions to its bounding surfaces. Here we consider these deformations and find the surface tractions required to bend a rectangular straight beam made of the fiber-reinforced hyperelastic material. By applying these surface tractions in the numerical solution of the problem with the developed subroutine, we will find stresses and deformations induced in the beam interior and compare the computed and the analytical solutions. We note that for this problem, deformations are inhomogeneous.

A schematic sketch of the problem studied is exhibited in Fig. 5, wherein both the rectangular and the cylindrical coordinate axes are shown. The finite plane strain bending of a rectangular beam is described by the deformation field 


\section{TIRE SCIENCE AND TECHNOLOGY}
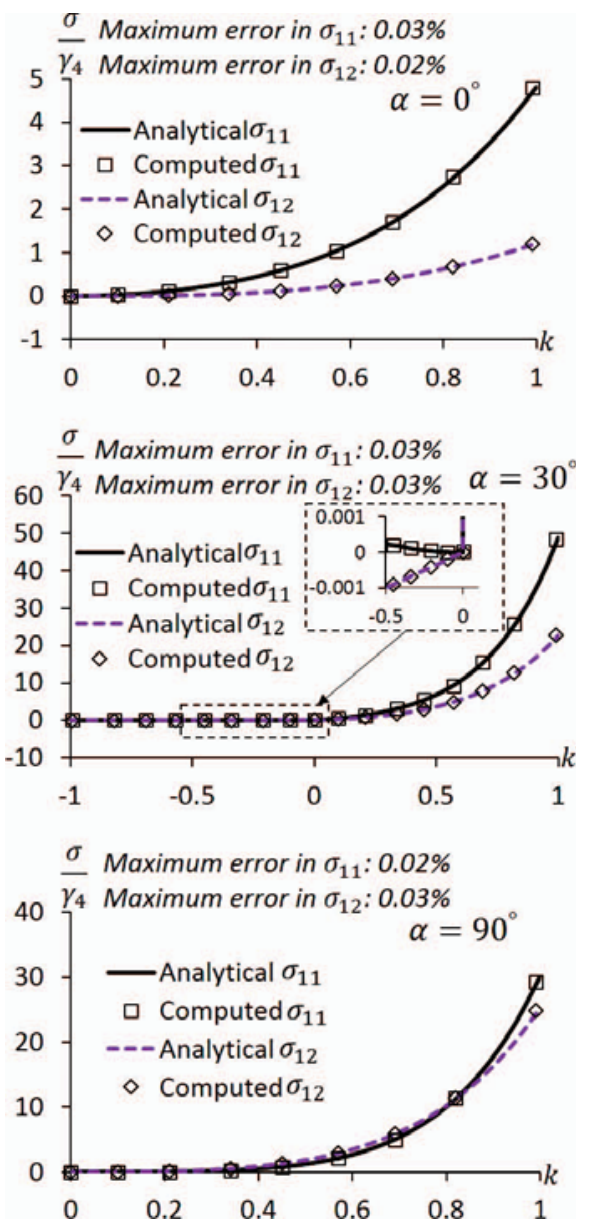

FIG. $4-$ For $\alpha=0^{\circ}, 30^{\circ}$, and $90^{\circ}$, normalized Cauchy stress components vs. the shear strain $\mathrm{k}$.

$$
r=\sqrt{2 A X_{1}+\beta}, \theta=D X_{2}, z=X_{3}
$$

where $A$ and $D$ are nonzero constants, $D=1 / A$ for deformations to be volume preserving, and the constant $\beta$ to be determined is related to the curvature of the deformed beam. The rectangular beam bounded by planes $X_{1}= \pm h$ and $X_{2}= \pm L$ in the reference configuration is deformed into the annular wedge bounded by the cylindrical surfaces $r=r_{1}$ and $r=r_{2}$ and the planes $\theta= \pm \theta_{0}$ in the current configuration. Planes $X_{2}=$ constant are deformed into planes $\theta=$ constant, and the planes $X_{1}=$ constant into radial surfaces $r=$ constant. 


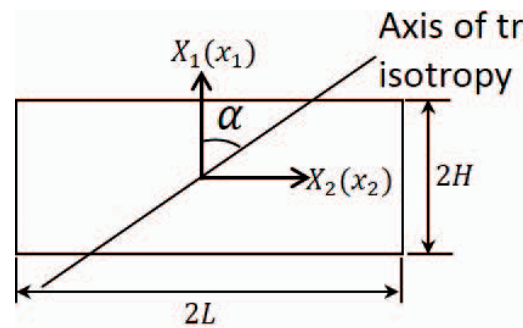

(1)

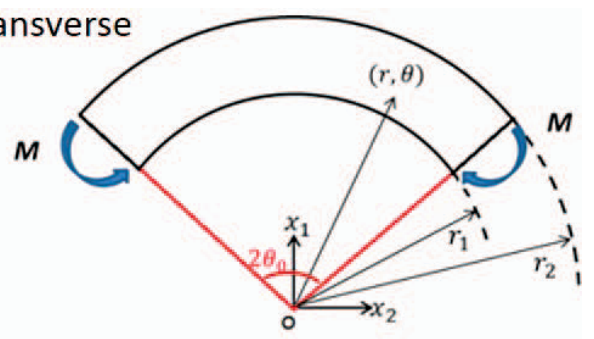

(2)

FIG. 5 - Schematic sketch of the bending of a straight rectangular beam into a circular beam; (1) reference configuration, (2) deformed configuration.

In rectangular Cartesian coordinates, the deformation [Eq. (24)] is given by

$$
x_{1}=\sqrt{2 A X_{1}+\beta} \cos \left(D X_{2}\right), x_{2}=\sqrt{2 A X_{1}+\beta} \sin \left(D X_{2}\right), x_{3}=X_{3}
$$

Referring the reader to Batra [23] and Lai et al. [29] for details, we have the following expressions for the physical components of tensors $\boldsymbol{B}$ and $\boldsymbol{C}$ in the cylindrical coordinate system.

$$
[B]=\left[\begin{array}{ccc}
\frac{A^{2}}{r^{2}} & 0 & 0 \\
0 & D^{2} r^{2} & 0 \\
0 & 0 & 1
\end{array}\right],[C]=\left[\begin{array}{ccc}
\frac{A^{2}}{r^{2}} & 0 & 0 \\
0 & D^{2} r^{2} & 0 \\
0 & 0 & 1
\end{array}\right]
$$

The strain invariants $I_{1}, I_{2}, I_{3}, I_{4}$, and $I_{5}$ are given by

$$
\begin{aligned}
& I_{1}=I_{2}=\frac{A^{2}}{r^{2}}+D^{2} r^{2}+1, I_{3}=1, I_{4}=\frac{A^{2}}{r^{2}}(\cos \alpha)^{2}+D^{2} r^{2}(\sin \alpha)^{2}, \\
& I_{5}=\frac{A^{4}}{r^{4}}(\cos \alpha)^{2}+D^{4} r^{4}(\sin \alpha)^{2}
\end{aligned}
$$

Substituting for $I_{1}, I_{2}, I_{4}$, and $I_{5}$ from Eqs. (25)—(27) into Eq. (12), we get the following expressions for the physical components of the Cauchy stress tensor in the cylindrical coordinate system.

$$
\begin{gathered}
\sigma_{r r}=-p+2\left(1-V_{f}\right)\left[C_{1} \frac{\boldsymbol{A}^{2}}{r^{2}}+C_{2}\left(1+\frac{\boldsymbol{A}^{2}}{r^{2}}\right)\right]+4 V_{f} H\left(I_{4}-1\right) \\
\left.\qquad \gamma_{4}\left(I_{4}-1\right) \frac{\boldsymbol{A}^{2}}{r^{2}}(\cos \alpha)^{2}+2 \gamma_{5}\left(I_{5}-1\right) \frac{\boldsymbol{A}^{4}}{r^{4}}(\cos \alpha)^{2}\right\}
\end{gathered}
$$




$$
\begin{aligned}
\sigma_{\theta \theta}= & -p+2\left(1-V_{f}\right)\left[C_{1} \frac{r^{2}}{\boldsymbol{A}^{2}}+C_{2}\left(1+\frac{r^{2}}{\boldsymbol{A}^{2}}\right)\right] \\
& +4 V_{f} H\left(I_{4}-1\right)\left\{\gamma_{4}\left(I_{4}-1\right) \frac{r^{2}}{\boldsymbol{A}^{2}}(\sin \alpha)^{2}+2 \gamma_{5}\left(I_{5}-1\right) \frac{r^{4}}{\boldsymbol{A}^{4}}(\sin \alpha)^{2}\right\} \\
\sigma_{z z}= & -p+2\left(1-V_{f}\right)\left[C_{1}+C_{2}\left(\frac{\boldsymbol{A}^{2}}{r^{2}}+\frac{r^{2}}{\boldsymbol{A}^{2}}\right)\right] \\
\sigma_{r \theta}= & 2 \sin 2 \alpha H\left(I_{4}-1\right)\left\{\left(V_{f} \gamma_{4}\right)\left(I_{4}-1\right)+2 V_{f} \gamma_{5}\left(I_{5}-1\right)\left(\frac{\boldsymbol{A}^{2}}{r^{2}}+\frac{r^{2}}{\boldsymbol{A}^{2}}\right)\right\} \\
\sigma_{z r}= & \sigma_{z \theta}=0
\end{aligned}
$$

For null body forces, equations expressing the balance of linear momentum in cylindrical coordinates are (see Lai et al. [29]):

$$
\begin{aligned}
\frac{\partial \sigma_{r r}}{\partial r}+\frac{1}{r} \frac{\partial \sigma_{r \theta}}{\partial \theta}+\frac{\partial \sigma_{r z}}{\partial z}+\frac{\sigma_{r r}-\sigma_{\theta \theta}}{r} & =0, \frac{\partial \sigma_{\theta r}}{\partial r}+\frac{1}{r} \frac{\partial \sigma_{\theta \theta}}{\partial \theta}+\frac{\partial \sigma_{\theta z}}{\partial z}+\frac{2 \sigma_{\theta r}}{r}=0 \\
\frac{\partial \sigma_{z r}}{\partial r}+\frac{1}{r} \frac{\partial \sigma_{z \theta}}{\partial \theta}+\frac{\partial \sigma_{z z}}{\partial z}+\frac{\sigma_{z r}}{r} & =0
\end{aligned}
$$

For $\alpha=0^{\circ}$ and $90^{\circ}, \sigma_{r \theta}=0$, and the analysis of the problem is simplified. For $\alpha$ $=0^{\circ}$ the presence of fibers only influences $\sigma_{r}$, and for $\alpha=90^{\circ}$ only $\sigma_{\theta \theta}$. We first study the problem for $\alpha=0^{\circ}$ and $90^{\circ}$. Substitution for stress components from Eq. (28) into Eq. (29) gives $\partial p / \partial z=0$ and $\partial p / \partial \theta=0$. Thus the hydrostatic pressure $p$ and hence all components of the stress tensor depend only upon the radial coordinate $r$. The integration with respect to $r$ of the only non-trivial equilibrium equation [Eq. (29)], gives

$$
\sigma_{r r}=\int_{r_{1}}^{r} \frac{\sigma_{\theta \theta}-\sigma_{r r}}{r} d r
$$

where we have used the boundary condition $\sigma_{r r}\left(r_{1}\right)=0$. The boundary condition $\sigma_{r r}\left(r_{2}\right)=0$ provides an equation for the determination of $p$ in terms of $A$ and $\beta$. The requirement that the resultant force on surfaces $\theta= \pm \theta_{0}$ vanishes gives

$$
\int_{r_{1}}^{r_{2}} \sigma_{\theta \theta} d r=0
$$


As mentioned above, the cylindrical surfaces $r=r_{1}$ and $r=r_{2}$ are also traction free. The moment, $M$, per unit length in the $X_{3}$-direction, applied at the end faces $\theta= \pm \theta_{0}$ required to bend the beam is given by

$$
\begin{aligned}
M & =\int_{r_{1}}^{r_{2}} r \sigma_{\theta \theta} d r \\
& =\int_{r_{1}}^{r_{2}}\left[r\left(\sigma_{\theta \theta}-\sigma_{r r}\right)+r \sigma_{r r}\right] d r \\
& =\int_{r_{1}}^{r_{2}} r\left(\sigma_{\theta \theta}-\sigma_{r r}\right) d r+\left.\frac{1}{2} r^{2} \sigma_{r r}\right|_{r_{1}} ^{r_{2}}-\int_{r_{1}}^{r_{2}} \frac{1}{2} r^{2} \frac{d \sigma_{r r}}{d r} d r \\
& =\int_{r_{1}}^{r_{2}} r\left(\sigma_{\theta \theta}-\sigma_{r r}\right) d r-\int_{r_{1}}^{r_{2}} \frac{1}{2} r^{2} \frac{d \sigma_{r r}}{d r} d r \\
& =\int_{r_{2}}^{r_{2}} r\left(\sigma_{\theta \theta}-\sigma_{r r}\right) d r-\int_{r_{1}} \frac{1}{2} r^{2} \frac{\sigma_{\theta \theta}-\sigma_{r r}}{r} d r \\
& =\int_{r_{1}}^{r_{2}} \frac{1}{2} r\left(\sigma_{\theta \theta}-\sigma_{r r}\right) d r
\end{aligned}
$$

where we have used the boundary conditions $\sigma_{r r}\left(r_{1}\right)=0, \sigma_{r r}\left(r_{2}\right)=0$, and Eq. (29). This simplification is also given in Truesdell and Noll [25]. Equations (31) and (32) relate $A$ and $\beta$ to the moment $M$ applied at the end faces.

For $\alpha$ other than $0^{\circ}$ and $90^{\circ}$, the hydrostatic pressure $p$ depends upon $\theta$, and equilibrium Eqs. (29) require that $\partial p / \partial \theta$ and $\partial p / \partial r$ are functions of $r$ only. Thus the condition $\partial^{2} p / \partial \theta \partial r=\partial^{2} p / \partial r \partial \theta$ for finding the pressure cannot be satisfied, and there is no solution of the problem of the type given by Eq. (25). However, the problem may have solutions other than those given by Eq. (25). Recall that Ericksen's theorem applies only to isotropic hyperelastic materials, and here we have a transversely isotropic material.

For infinitesimal deformations one defines the neutral surface by using Eq. (31), and it passes through the centroid of the beam cross-section. Both the hoop strain and the hoop stress vanish at points on the neutral surface. However, for the nonlinear theory, it need not pass through the beam centroid. Furthermore, points where $\sigma_{\theta \theta}=0$ and $\varepsilon_{\theta \theta}=0$ need not coincide with each other. Here $\varepsilon_{\theta \theta}$ is a component of the Almansi-Hamel strain, $\boldsymbol{\varepsilon}=1 / 2\left(\mathbf{1}-\boldsymbol{B}^{-1}\right)$, in the $\theta$-direction. 
68 TIRE SCIENCE AND TECHNOLOGY
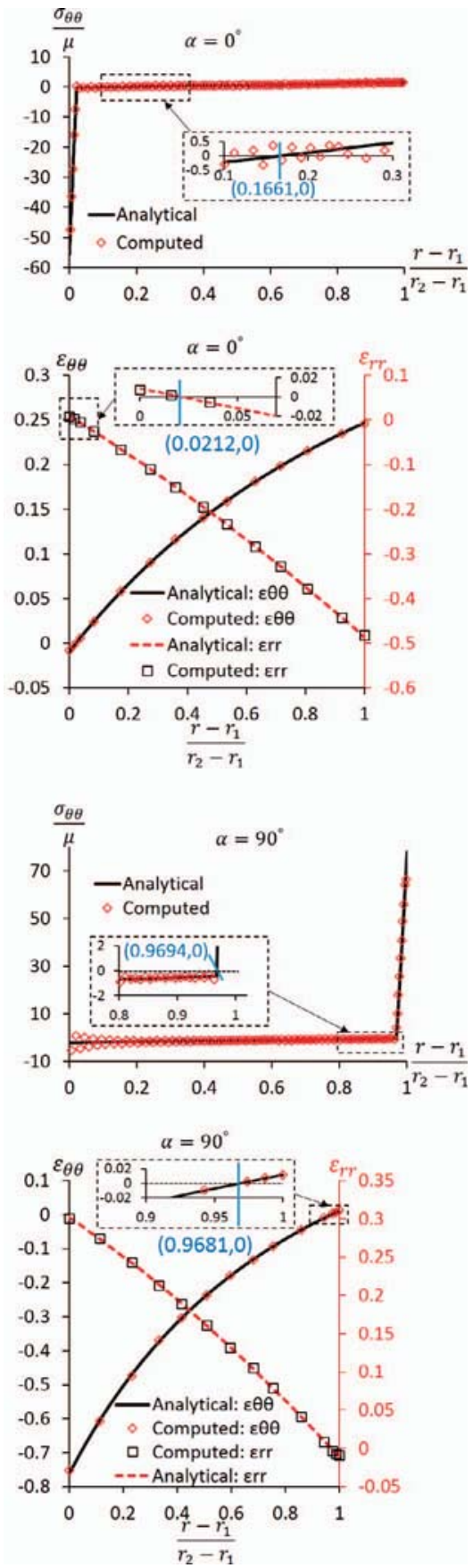
While numerically analyzing the plane strain boundary value problem for $\alpha$ $=0^{\circ}$ and $90^{\circ}$, we study deformations of only the right half of the beam, since the beam geometry and its deformations are symmetric about the plane $X_{2}=0$. However, for other values of $\alpha$, deformations may not be symmetric because the material of the beam is transversely isotropic. Boundary conditions used are

$$
\begin{aligned}
& \text { Surface }: X_{1}=-h: u_{1}=x_{1}-X_{1}, u_{2}=x_{2}-X_{2} \\
& \text { Surface }: X_{1}=h: \sigma_{r r}=\sigma_{r \theta}=0 \\
& \text { Surface }: X_{2}=0: u_{1}=x_{1}-X_{1}, u_{2}=0 \\
& \text { Surface }: X_{2}=L: u_{1}=x_{1}-X_{1}, u_{2}=x_{2}-X_{2}
\end{aligned}
$$

We set the $X_{3}$-displacement of all nodes equal to zero to simulate plane strain deformations. The displacements given by Eq. (33) are incrementally applied on the faces $X_{1}=-h, X_{2}=0$, and $X_{2}=L$ and are simultaneously incremented by the same percentage till their final values. We note that $x_{1}$ and $x_{2}$ are calculated from Eq. (25) after finding values of constants $A$ and $\beta$ from the applied moment, $M$. For the example problems studied below, we have used values of material parameters listed just before the section "Simple Extension of a Cube" and have set $L=2 \mathrm{~h}=$ $100 \mathrm{~mm}$. For $\alpha=0^{\circ}$, values of other parameters are $A=0.2018 \mathrm{~m}, \beta=0.0602 \mathrm{~m}^{2}$ that correspond to $M=11.94 \mathrm{kN}-\mathrm{m}$, and $\theta_{0}=28.4^{\circ}$, and for $\alpha=90^{\circ}$, we have $\mathrm{A}=$ $0.318 \mathrm{~m}, \beta=0.0718 \mathrm{~m}^{2}$, which correspond to $M=43.78 \mathrm{kN}-\mathrm{m}$ and $\theta_{0}=18.02^{\circ}$.

We discretize the $100 \mathrm{~mm} \times 100 \mathrm{~mm} \times 1 \mathrm{~mm}$ region in the reference configuration occupied by the right half of the beam into $110 \times 150 \times 1 \mathrm{FE}$ mesh of eight-node brick elements. As mentioned above, we have set $K=150 \gamma_{4}$ in order to save on computational resources. Thus the incompressibility condition is not well satisfied and the computed hydrostatic pressure exhibits oscillations whose amplitude depends upon the value of $K$ and the FE mesh used. For $\alpha=0^{\circ}$ and $90^{\circ}$ the distribution of the computed Cauchy hoop stress normalized by the shear modulus, $\mu=2\left(C_{1}+C_{2}\right)$, and the Almansi-Hamel hoop strain are compared with their values from the analytical solution in Fig. 6. The two sets of results differ by at most $4.9 \%$. When the FE mesh was refined to $205 \times 150 \times 1$ elements, the maximum difference between the computed and the analytical solutions was reduced to $3.8 \%$, mainly because of the improvement in the computed values of the hydrostatic pressure. However, the CPU time required to solve the problem increased by a factor of 10 . The effect of different responses of fibers when they are deformed in tension and compression should be clear from the plots of Fig. 6. In the region where fibers are stretched, stresses induced are much larger than those in the region where fibers are compressed because of the huge difference (a factor of 1000) in the axial modulus of the fibers in tension and compression. In order for the resultant axial force on a cross-section to be zero, a much larger portion of the material in the radial direction is experiencing compressive hoop stress than that for which the hoop stress is tensile. The radial locations of points where $\sigma_{\theta \theta}=0$ and $\varepsilon_{\theta \theta}=0$ are different because the hydrostatic pressure makes a

FIG. $6-$ For $\alpha=0^{\circ}$ and $90^{\circ}$, the through-the-thickness variation of the hoop stress and the AlmansiHamel hoop strain. 


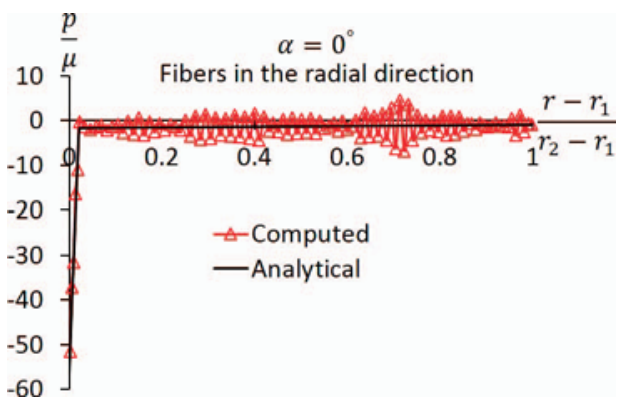

FIG. 7. - For $\alpha=0^{\circ}$, the through-the-thickness variation of the hydrostatic pressure.

significant contribution to the radial and the hoop stresses. For $\alpha=0^{\circ}, \sigma_{\theta \theta}=0$, and $\varepsilon_{\theta \theta}=0$ at points situated, respectively, at non-dimensional radii of 0.1661 and 0.0212 from the inner surface. However, the hoop stress at points for which the non-dimensional radius exceeds 0.0212 is miniscule as compared to its maximum magnitude of 55. We note that at points for which the non-dimensional radius is less than $0.0212, \sigma_{\theta \theta}<0$ even though $\varepsilon_{\theta \theta}>0$ because of $\varepsilon_{\mathrm{rr}}<0$ and the significant contribution from the hydrostatic pressure.

The hydrostatic pressure distribution exhibited in Fig. 7 for $\alpha=0^{\circ}$ is oscillatory due to a small value assigned to the bulk modulus $K$. Oscillations in the hydrostatic pressure cause oscillations in the Cauchy stresses even though strains vary smoothly in the radial direction. As mentioned above, a larger value of $K$ could not be used because of the excessive CPU time required to compute results. The oscillations in the hydrostatic pressure for $\alpha=90^{\circ}$ are of larger amplitude than those for $\alpha=0^{\circ}$. However, the amplitude of oscillations is less than $5 \%$ of the maximum values of stresses induced in the deformed beam. Ideally, one should compute results with successively refined FE meshes and several values of the bulk modulus to obtain converged results. Unfortunately, this could not be accomplished due to constraints of time and the computational resources. Oscillations in the hydrostatic pressure can be smoothened out by using a pressure smoothing technique (e.g., see Hughes [30]) such as averaging the pressure in two consecutive elements, but it has not been implemented in the subroutine.

Results for values of $\alpha$ other than $0^{\circ}$ and $90^{\circ}$ have not been computed because there is no analytical solution available with which computed results could be compared. If we were to deform such a rectangular beam by applying only moments on the faces $\mathrm{X}_{2}= \pm \mathrm{L}$, the beam will not be bent into a circular arc, and the faces into which surfaces $\mathrm{X}_{1}= \pm h$ are deformed may not be smooth. A difficulty in applying pure moments at the end faces is determining the distribution of normal surface tractions on them.

\section{Conclusions}

By assuming that a fiber-reinforced rubberlike material can be modeled as a homogeneous and transversely isotropic hyperelastic material with the fiber direction as the axis of transverse isotropy, we have proposed an expression for 
the strain energy density in terms of the five invariants of the right Cauchy-Green strain tensor. For compressible and incompressible materials it involves five and four material constants, respectively. However, the hydrostatic pressure for incompressible materials is found from the solution of the boundary value problem and is thus an independent variable along with the three displacement components. The material model represents a different response for axial compression and axial tension along the fiber direction. The constitutive relation has been implemented in the commercial software, LS-DYNA, as a user defined subroutine. For three static boundary value problems, results computed by using the subroutine have been found to agree well with those computed analytically. Full verification of the implementation of the subroutine requires similar comparisons for numerous boundary value problems. Also, predictions from the material model need to be compared with the test data to validate it and establish its range of applicability. When test data for different modes of deformation become available, the form of the strain energy density assumed here may need to be modified. However, the new form of the strain energy density can be similarly implemented in LS-DYNA.

\section{Acknowledgments}

This work was supported by a grant from the Center for Tire Research (an NSF Industry/University Cooperative Research Center). We are indebted to the industry mentors for providing suggestions to improve upon the work.

\section{References}

[1] Batra, R. C., "Rubber Covered Rolls, The Nonlinear Elastic Problem," Journal of Applied Mechanics, Vol. 47, 1980, pp. 82-86.

[2] Bapat, C. N. and Batra, R. C., "Finite Plane Strain Deformations of Nonlinear Viscoelastic Rubber Covered Rolls," International Journal for Numerical Methods in Engineering, Vol. 20, 1984, pp. 1911-1927.

[3] Helnwein, P., Liu, C. H., Meschke, G., and Mang, H. A., "A New 3-D Finite Element Model for Fiber-Reinforced Rubber Composites-Application to Analysis of Automobile Tires," Finite Element Analysis and Design, Vol. 14, 1993, pp. 1-16.

[4] Meschke, G. and Helnwein, P., "Large Strain 3D Analysis of Fiber-Reinforced Composites Using Rebar Elements: Hyperelastic Formulations for Fiber," Computational Mechanics, Vol. 13, 1994, pp. 241-254.

[5] Mang, H. A. and Meschke, G., "Finite Element Analysis of Reinforced and Prestressed Concrete Structures," Engineering Structures, Vol. 13, 1991, pp. 211-226.

[6] ABAQUS, ABAQUS Version 6.11 Documentation, ABAQUS, Inc., Sunnyvale, CA, 2011.

[7] Batra, R. C., Gopinath, G. and Zheng, J. Q., "Material Parameters for Pressure-Dependent Yielding of Unidirectional Fiber-Reinforced Polymeric Composites," Composites Part B, Vol. 43, 2012, pp. 2594-2604.

[8] Pipkin, A. C., "Stress Analysis for Fiber-Reinforced Materials," Advances in Applied Mechanics, Vol. 19, 1979.

[9] Spencer, A. J. M., "Constitutive Theory of Strongly Anisotropic Solids," in Spencer, A. J. M. (ed.), Continuum Theory of the Mechanics of Fiber-Reinforced Composites, Springer, Vienna, 1984, pp. 1-32. 


\section{TIRE SCIENCE AND TECHNOLOGY}

[10] Qiu, G. Y. and Pence, T. J., "Remarks on the Behavior of Simple Directionally Reinforced Incompressible Nonlinearly Elastic Spheres," Journal of Elasticity, Vol. 49, 1997, pp. 1-30.

[11] Merodio, J. and Ogden, R. W., "Mechanical Response of Fiber-Reinforced Incompressible Nonlinear Elastic Solids," International Journal of Nonlinear Mechanics, Vol. 40, 2005, pp. 213-227.

[12] Holzapfel, G. A., Gasser, T. C., and Ogden, R. W., "A New Constitutive Framework for Arterial Wall Mechanics and a Comparative Study of Material Models," Journal of Elasticity, Vol. 61, 2000, pp. 1-48.

[13] Gasser, T. C., Ogden, R. W., and Holzapfel, G. A., "Hyperelastic Modelling of Arterial Layers with Distributed Collagen Fibre Orientations," Journal of the Royal Society Interface, Vol. 3, 2006, pp. 15-35.

[14] deBotton, G., Hariton, I., and Socolsky, E. A., "Neo-Hookean Fiber-Reinforced Composites in Finite Elasticity," Journal of the Mechanics and Physics of Solids, Vol. 54, 2006, pp. 533-559.

[15] Guo, Z. Y., Peng, X. Q., and Moran, B., "A Composites-Based Hyperelastic Constitutive Model for Soft Tissue with Application to the Human Annulus Fibrosus." Journal of the Mechanics and Physics of Solids, Vol. 54, 2006, pp. 1952-1971.

[16] Weiss, J. A., Maker, B. N., and Govindjee, S., "Finite Element Implementation of Incompressible, Transversely Isotropic Hyperelasticity," Computer Methods in Applied Mechanics and Engineering, Vol. 135, 1996, pp. 107-128.

[17] Peng, X. Q., Guo, Z. Y., and Moran, B., "An Anisotropic Hyperelastic Constitutive Model with Fiber-Matrix Shear Interaction for the Human Annulus Fibrosus," Journal of Applied Mechanics, Vol. 73, 2006, pp. 815-824.

[18] Ericksen, J. L. and Rivlin, R. S., "Large Elastic Deformations of Homogeneous Anisotropic Materials," Archive for Rational Mechanics and Analysis, Vol. 3, 1954, pp. 281-301.

[19] Batra, R. C., "Universal Relations for Transversely Isotropic Elastic Materials," Mathematics and Mechanics of Solids, Vol. 7, 2002, pp. 421-437.

[20] Batra, R. C. and Liang, X. Q., "Finite Dynamic Deformations of Smart Structures," Computational Mechanics, Vol. 20, 1997, pp. 427-438.

[21] Love, B. M. and Batra, R. C., "Determination of Effective Thermomechanical Parameters of a Mixture of Two Thermoviscoplastic Constituents," International Journal of Plasticity, Vol. 22, 2006, pp. 1026-1061.

[22] Bowen, R. M., "Theory of Mixtures," in Eringen A. C. (ed.), Continuum Physics, Vol. 3, Academic Press, New York, 1976.

[23] Batra, R. C., Elements of Continuum Mechanics, AIAA Publishers, Reston, VA, 2006.

[24] Mooney, M. A., "Theory of Large Elastic Deformations," Journal of Applied Physics, Vol. 11, 1940, pp. 582-592.

[25] Truesdell, C. and Noll, W., "Nonlinear Field Theories of Mechanics," Handbuch der Physik, Vol. 3, 1965, p. 3.

[26] Livermore Software Technology Corporation, LS-DYNA R7.1 Keyword User's Manual, Appendix A, LSTC, Livermore, CA, 2014.

[27] Belytschko, T. and Bindeman, L. P., "Assumed Strain Stabilization of the Eight Node Hexahedral Element," Computer Methods in Applied Mechanics and Engineering, Vol. 105, 1993, pp. 225-226.

[28] Ericksen, J. L., "Deformations Possible in Every Isotropic Incompressible Perfectly Elastic Body," Zeitschrift für angewandte Mathematik und Physik, Vol. 5, 1954, pp. 466-486.

[29] Lai, W. M., Rubin, D., and Krempl, E., Introduction to Continuum Mechanics, 4th ed., Elsevier, Philadelphia, 2010.

[30] Hughes, T. J. R., "The Finite Element Method: Linear Static and Dynamic Finite Element Analysis," Dover Civil and Mechanical Engineering, Mineola, NY, 2000. 